\title{
Yellow nail syndrome
}

\author{
Allie Preston, BS ${ }^{\mathrm{a}}$ (D), Kirstin Altman, MD ${ }^{\mathrm{b}}$ (D), and Gregory Walker, MD \\ ${ }^{a}$ Texas A\&M College of Medicine, Temple, Texas; ${ }^{b}$ Department of Dermatology, Scott \& White Medical Center, Temple, Texas
}

\begin{abstract}
Yellow nail syndrome is a rare condition that presents as a clinical triad of chronic respiratory symptoms, lymphedema, and yellow, dystrophic nails. We present the case of a patient with yellow nail syndrome who had yellow, thickened nails unresponsive to antifungal treatment with concomitant lower-extremity lymphedema and a negative pulmonary workup despite chronic cough. His nails responded well to treatment with vitamin $E$ and pulsed fluconazole.
\end{abstract}

KEYWORDS Dystrophic nails; lymphedema; respiratory complications

Y ellow nail syndrome (YNS) is a rare condition characterized by a clinical triad of slow-growing, yellow, dystrophic nails, lymphedema, and recurring respiratory symptoms. ${ }^{1}$ It typically presents between the fourth and eighth decades with a slight male predominance, and the greatest incidence occurs after the age of $50 .^{2}$ YNS was first described in 1964 , and fewer than 400 cases have been reported since. ${ }^{2-4}$ The diagnosis of YNS is made when two out of three characteristic symptoms, including nail changes, lymphedema, and respiratory symptoms, are present. Only one-third of patients with this condition have all three symptoms simultaneously, and occasionally the individual symptoms may appear alone and sequentially, which can make diagnosis difficult. ${ }^{2,4,5}$

\section{CASE DESCRIPTION}

A 39-year-old man with no significant past medical history presented to our outpatient dermatology clinic with a 1-year history of slowly growing, yellow fingernails (Figure 1a) and toenails. Upon review of systems, he also reported a history of progressive, chronic cough. At the time of presentation, he had recently completed a 6-month course of daily terbinafine but had no improvement of his nail symptoms. Physical exam showed yellow discoloration of all nails with subungual hyperkeratosis. Of note, nonpitting edema was present in the bilateral lower extremities. Histopathologic evaluation of nail clippings was negative for fungal organisms.

Due to clinical suspicion for YNS and his reported history of cough, the patient was referred to pulmonology to evaluate respiratory complications. Chest $\mathrm{x}$-ray was negative for any associated pulmonary pathology. However, because of the nail findings and the refractory lower-extremity lymphedema, he still met sufficient criteria for diagnosis of YNS. Thus, the patient was started on pulsed fluconazole and vitamin $\mathrm{E}$ for treatment of his nail symptoms, and at follow-up 2 months later, he had significant improvement in his nails (Figure 1b). In addition to treatment of his nail symptoms, he was also referred for lymphedema therapy and will continue to follow up with pulmonology yearly to monitor for the development of associated respiratory complications.

\section{DISCUSSION}

The presentation of yellow nails is often the most striking feature of YNS. Nail changes can affect either the fingernails, toenails, or both; they include yellowing, slow growth, onycholysis, inadequate cuticles, and cross-ridging. ${ }^{6}$ By definition, fungal cultures of affected nails are negative and therefore they will not respond to treatment with antifungals alone. ${ }^{5}$

Pleural effusion (46\%), bronchiectasis (44\%), and chronic sinusitis (41\%) are among the most common associated respiratory disorders, but occasionally the only symptom is cough. ${ }^{5}$ Pleural effusion is frequently bilateral $(68.3 \%$ of patients in one study) and typically exudative with predominant lymphocytosis. ${ }^{2,5,7,8}$ When present, pulmonary pathology can be quite severe; fortunately, despite his chronic cough, our patient had not experienced any significant pulmonary complications, but he will be followed closely.

Corresponding author: Allie Preston, BS, Texas A\&M College of Medicine, 2401 S. 31st St., Medical Education Building, $407 \mathrm{H}$, Temple, TX 76508 (e-mail: apreston@medicine.tamhsc.edu)

Color versions of one or more of the figures in this article can be found online at www.tandfonline.com/umbc.

Received April 29, 2018; Revised May 31, 2018; Accepted June 7, 2018. 

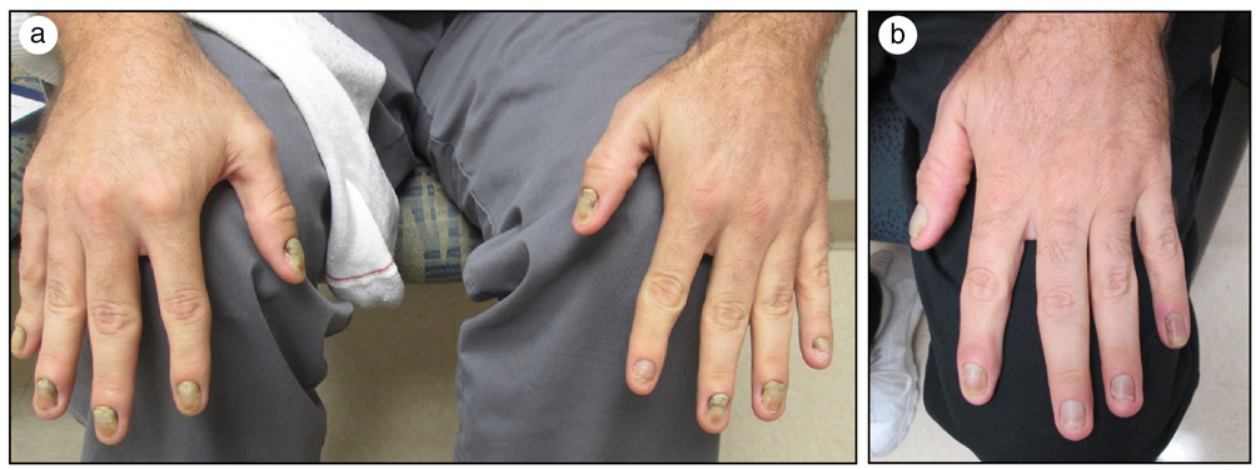

Figure 1. Fingernails (a) pretreatment and (b) posttreatment.

Lymphedema, most commonly affecting the bilateral lower extremities, is also frequently seen in patients with YNS and may be the first sign of disease in about a third of cases. ${ }^{4}$ In one study, all of the patients who had pleural effusions also had lymphedema, but in another the reported presence of lymphedema ranged from $29 \%$ to $80 \%{ }^{2,4}$ The variations regarding reported rates of symptomology in YNS are likely skewed by the specialist to whom the patient initially presents as well as the time interval (ranging from several months to many years) that often exists between the first symptoms and the subsequent diagnostic ones. ${ }^{4,8}$

The etiology of YNS is poorly understood but is thought to involve abnormalities in anatomic or functional lymphatic drainage. ${ }^{1,3,5,8,9}$ Increased microvascular permeability and subsequent protein leakage would also account for its common association with hypoalbuminemia and high albumin levels within pleural fluid. ' Cases of familial YNS have been reported, but it is much more likely to be an acquired rather than inherited condition. ${ }^{6}$ An association with autoimmune diseases, such as rheumatoid arthritis, autoimmune thyroiditis, and systemic lupus erythematosus; connective tissue diseases; neoplasms; and certain drugs has also been suggested, but a definitive relationship has not yet been established. ${ }^{2,5,7}$ Because the underlying pathophysiology of YNS is not completely understood, it remains a clinical diagnosis of exclusion. ${ }^{2,6}$

There is no consensus therapy for the treatment of YNS, but a variety of case studies indicate possible effective treatment options typically targeted at the patient's specific symptoms. ${ }^{6}$ Over time, nail symptoms will resolve in many patients without treatment, but high-dose vitamin E supplementation (both topically and orally) has demonstrated some clinical benefit - perhaps due to its antioxidant properties. ${ }^{4-7}$ Oral antifungals, such as itraconazole or fluconazole, in combination with vitamin $\mathrm{E}$ have been beneficial for treating the nail symptoms of YNS in some cases. ${ }^{4,6}$ Although the disease is not caused by fungal organisms, the azole class of antifungals is believed to stimulate growth of the nail to enhance outgrowth and clearance of the nail discoloration. ${ }^{4}$

Octreotide has been used for the medical management of YNS-associated pleural effusions due to its ability to inhibit lymphatic secretion. ${ }^{6}$ However, the most effective treatments for pleural effusions in patients with YNS appear to be pleurodesis or decortication/pleurectomy; therapeutic thoracentesis is rarely effective due to reoccurrence of the effusion., ${ }^{2,6}$ Antibiotics and bronchodilators may also be beneficial, especially in patients with bronchiectasis and recurrent respiratory infections. ${ }^{6}$ Compression stockings and diuretics are frequently used in patients with edema and offer mild benefits, but surgery with excision of the edematous subcutaneous tissue is sometimes recommended in more refractory cases. ${ }^{5,6}$ Overall, the prognosis for patients with YNS is favorable, and, despite patients with YNS having a shorter life expectancy than the general population, no deaths linked directly to YNS have been reported. ${ }^{5,6}$ This case highlights an interesting condition that should be included in the differential diagnosis of patients with dystrophic, yellow nails, especially when fungal cultures are negative and the condition is not responding to treatment with antifungals alone.

\section{ORCID}

Allie Preston (iD http://orcid.org/0000-0001-7019-4830

Kirstin Altman (DD http://orcid.org/0000-0002-4399-5090

1. Emerson PA. Yellow nails, lymphoedema, and pleural effusions. Thorax. 1966;21(3):247-253.

2. Valdés L, Huggins JT, Gude F, et al. Characteristics of patients with yellow nail syndrome and pleural effusion. Respirology. 2014;19(7): 985-992. doi:10.1111/resp.12357.

3. Samman PD, White WF. The yellow nail syndrome. Br J Dermatol. 1964;76:153-157.

4. Vignes S, Baran R. Yellow nail syndrome: a review. Orphanet J Rare Dis. 2017;12(1):42-52. doi:10.1186/s13023-017-0594-4.

5. Maldonado F, Tazelaar HD, Wang C-W, Ryu JH. Yellow nail syndrome: analysis of 41 consecutive patients. Chest. 2008;134(2): 375-381. doi:10.1378/chest.08-0137.

6. Kurin M, Wiesen J, Mehta AC. Yellow nail syndrome: a case report and review of treatment options. Clin Respir J. 2017;11(4):405-410. doi:10.1111/cri.12354.

7. Banerjee A, Kanti-Biswas A, Bala S, Ghosh A. Yellow nail syndrome: a rare entity. Indian Dermatol Online J. 2014;5(4):529-531. doi:10.4103/2229-5178.142551.

8. Hiller E, Rosenow EC, Olsen AM. Pulmonary manifestations of the yellow nail syndrome. Chest. 1972;61(5):452-458.

9. Alessandro A, Muzi G, Monaco A, Filiberto S, Barboni A, Abbritti G. Yellow nail syndrome: does protein leakage play a role? Eur Respir J. 2001;17(1):149-152. 